\title{
Power-line communication between parallel DC-DC optimizers on a high voltage direct current bus
}

\author{
T. V. Nguyen, P. Petit, F. Maufay, M. Aillerie \& J. P. Charles \\ Lorraine University, Metz, France
}

\begin{abstract}
This contribution presents one developed solution for parallel photovoltaic energy generators based on a distributed architecture with individual DC-DC converters, named optimizers, connected to an intermediary high voltage direct current (HVDC) bus. This power bus also supports the communication between optimizers and a central controller using the power-line communication (PLC) approach. Then the optimizers are optimized for an interface output stage assuming the PLC on the HVDC bus. This work, from the conception to the realization of a prototype, concerns the analysis of the nature of the pertinent information to the exchange between the converter and the central controller. The information can include the $\mathrm{I}-\mathrm{V}$ characteristics and the temperature of the individual sources. The development of an electronic prototype that integrates recently available components is made and the technological constraints at the possible available frequency bandwidth are considered. The concrete realization will be presented in fine.
\end{abstract}

Keywords: power-line communication (PLC), high voltage direct current (HVDC), smart DC-DC converter, optimizer, renewable energy.

\section{Introduction}

The management and optimization of conversion and distribution of electrical energy generated from renewable sources, such as solar and wind power is a technology key-lock to improve the performance and the stability of the overall energy transfer systems. The distributed architecture of energy sources integrating smart grid concepts is a new approach allowing the individual optimization of the production from each source. This approach requires the 
adjunction in the system of intra and inter-communication tools with a central controller. One technical solution is PLC using the existing power-line infrastructure for communication purposes. This new possibility of management allows improving performance and stability in the overall energy transfer process. Unfortunately, the majority of recent contributions have discussed PLC for high data-rate applications like Internet access or multimedia communication serving a relatively small number of users, and only few works concern PLC for slow rate communication and high multipoint systems.

In recent years, some new energy power supply concepts have been introduced. One of them newly introduced in renewable energy systems is the smart grid concepts [1]. Moreover, nowadays power-engineering domain is facing enormous challenges since the increasing interest in intermittent renewable energies impose a completely new operation paradigm. The use of these resources must be done in ways that guarantee a secure, autonomous, sustainable, clean, and competitive operation. In a smart grid approach, distributed energy resources are interconnected to be able to provide sufficient and continuous energy. Dimensioning is done to provide energy as a significant part of the consumption demand. Many of these sources and applications are of DC type, and can be followed by a converter DC-ac stage. With the strong incentives of green and free energy sources and advancement in storage technology, power generation in a smart grid approach is not considered to be connected only to a distribution grid with coal-fired or gas-fired power plants, but can also constitute a stand alone installation for anyone who wishes to produce its own electricity.

\begin{tabular}{|ll|}
\hline \multicolumn{2}{l|}{ Nomenclature } \\
C & Capacitors (nF) \\
D1, D11 & Schottky Diodes \\
HVDC & High Voltage Direct Current \\
Ls, Lp1, Lp1 & Inductors $(\mathrm{mH})$ \\
M, M11 & MOSFET switch \\
n1, n2, n3 & Principal and secondary inductors $(\mathrm{mH})$ \\
MPPT & Maximum Power Point Tracker \\
MV & Medium-voltage \\
PIC & Peripheral Interface Controller \\
PWM & Pulse Width Modulation \\
PLC & Power-line Communication \\
R & Resistors $(\Omega)$ \\
\hline
\end{tabular}

Data transmission is also required in the proposed smart grid concept based on HVDC distribution system from renewable sources. Data transmission is needed for the required functionalities and applications that will be integrated into the system, for example for parallel DC-DC converter monitoring with power outage management and protection. For this purpose, PLC is a feasible alternative. 
One possible response to these challenges can be the solution proposed in Fig. 1. In this figure, we present a power supply system composed with various sources such as solar cells with possible different technologies, wind turbines, and compressed air energy storage (CAES) system or any other DC electric sources interconnected by a high voltage DC bus; As a result of such solution is a global generator implying a small wire section for the internal bus, and a global relative low cost architecture [2,3]. This solution implies that the sources deliver energy through the HVDC bus via individual high step-up DC-DC optimizers. In case of ac distribution, a central DC-ac inverter interfaces the HVDC bus to the grid. Thus, the HVDC system is designed to replace both traditional mediumvoltage (MV) overhead line branches and low-voltage ac distribution by a highvoltage DC distribution system. It is to be noted that, in this distributed configuration, maximum power point trackers (MPPT) need to be integrated in each individual converter. Additionally, as these MPPTs optimize the power available from each individual source, the global efficiency of the system will be higher than in case of a central architecture. Currently, in numerous industrial domains, most of the distributed electricity generation and storage systems use DC voltage and HVDC systems are intensively studied and developed for changing the electric distribution from ac system to DC one. It is to be of note that an additional advantage of DC voltage for smart grids is that no synchronization is required for distributed generation.

In an AC production system integrating an intermediary HVDC bus, the quality of the ac low-voltage at the output of the generator can be improved with a customer inverter by the reduction of the number of branches of the MV grid and a diminution of the length of the lines. The overhead lines can be replaced by underground low-voltage cables. This directly results in a decrease of the amount of faults generally occurring in MV grids. Thus, the quality and reliability of the electricity distribution is improved, and the costs caused by power outages reduced. The HVDC bus mainly defines the structure and dimensions of the proposed communication architecture when the communication is implemented with PLC. Thus, the structure and dimensions of the HVDC bus, linked to a physical geographic area, bring challenges to PLC, such as the reliability of the communication range, as for example, the determination of the PLC repeater power inserted in the bus. In addition, converters in the bus generate harmonics and interferences to the channel. These, combined with challenges among the implemented functions in the grid, set the boundary conditions and the minimum requirements for PLC.

Thus, we can summarize that the main objectives in developing distribution systems and pursuing for smart grids are the cost efficiency and the reliability of the electricity distribution. Ubiquitous communication plays a key role in smart grids and the HVDC concept presents a novel approach to implement a smart grid [4].

In this work, based on a set of requirements and measurements, PLC architecture is proposed for HVDC systems. It uses a commercial PLC technique and dedicated signal coupling method. The suitability of the proposed network for HVDC system is assessed by its compliance with the requirements set for this 


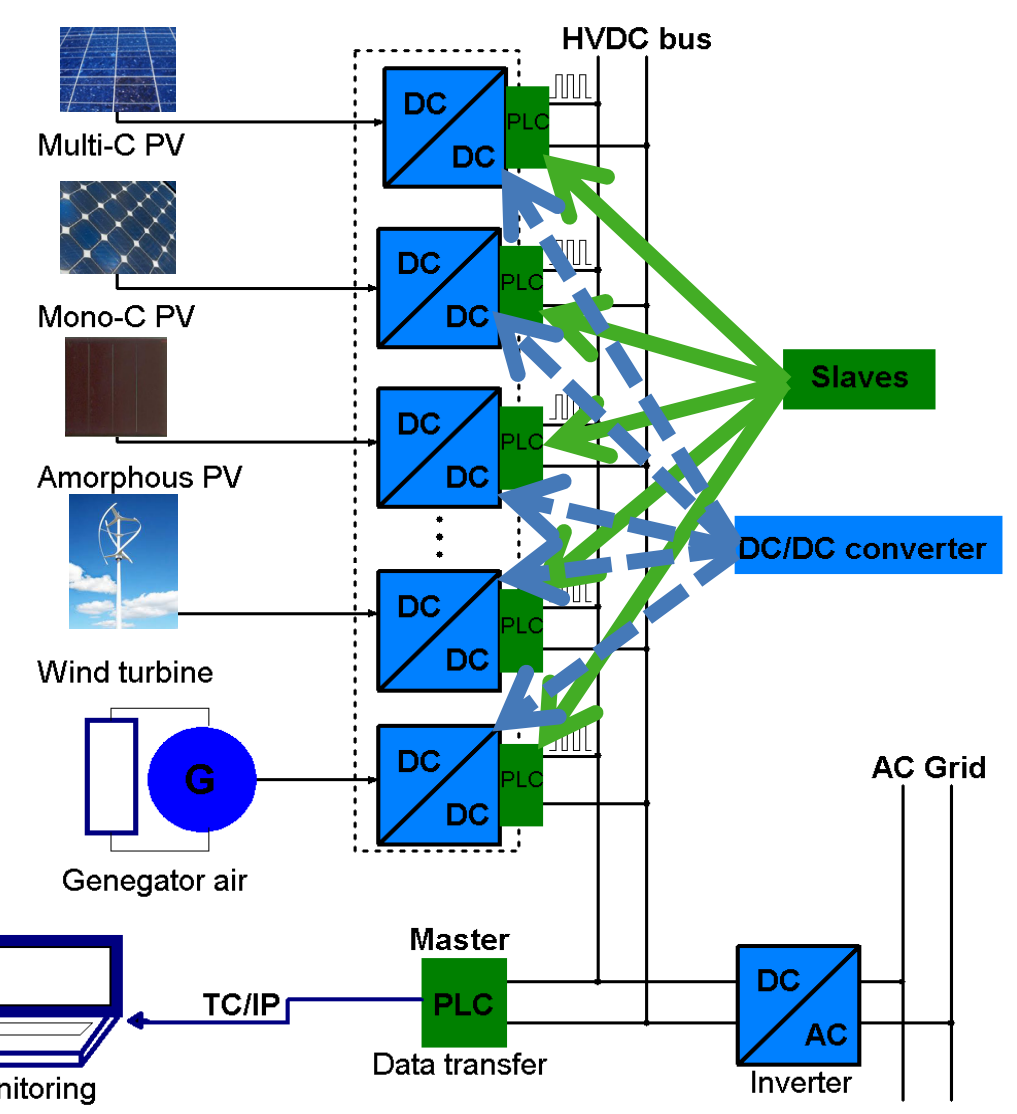

Figure 1: $\quad$ Schematic of a parallel structure of high voltage DC bus with PLC system on HVDC bus.

theoretical analysis and practical data transmission tests. We present a simple hardware implementation of a distributed architecture based on smart DC-DC optimizers integrating the power conversion and interface communication stages. PLC-based network architecture for the HVDC bus system that meets all the requirements presented above is described. It is to be noted that a self-power supply stage can also be added in the optimizer, but only PLC developments will be presented in this contribution; the main energy converter functions, with their MPPT algorithms and the self-power stage are presented in other contributions [5-8].

The PLC system is connected to power-lines of the HVDC bus using proper interfacing circuits, which are used to provide electrical isolation and impedance adaptation between the power DC-DC conversion part of the converter and the power-line network. This means that the PLC systems can be considered as an 
additional part of each converter, without modification of its basic structure. Nevertheless, theses two main stages are driven by a common Peripheral Interface Controller (PIC) microcontroller assuming both the functions of tracker and PLC master-slave controller. The system is suitable for data communications within a local power network area, such as remote automatic meter reading, f re and security alarm control. The system is built using digital modulation [9] to reduce the complexity of the overall DC-DC converter-PLC controller. All these simple concepts, mainly the two independent stages and a common control yield to a great reduction in the cost of the overall system.

\section{Communication requirements for PLC system on HVDC bus for renewable energy generator}

A distributed energy generator, associated with an energy transfer by intermediate HVDC bus, integrating smart grid concept system contains functionalities, such as smart metering, grid protection, control, and monitoring implemented with communication between the parallel DC-DC optimizer and the HVDC bus [10]. Different functions set different requirements for the communications. Other functionalities, especially grid protection and monitoring, include requirements for latency and jitter. Smart metering applications, in turn, requires a for throughput potential, especially in medium and small grids with many sources, where the total amount of transmitted data can be up to kilobytes. Thus, broadband data rate $(>1 \mathrm{kbps})$ is one of the requirements. In addition, the number of functionalities will increase in the future, such as, the control of distributed power production units and management of energy storages. This has also to be taken into account when considering the communication method or the selected protocol on the HVDC bus. Other requirements for communication besides sufficient latency and data rate are the reliability and range of the communication. Functions such as grid protection is physically located and executed at the grid level. With PLC, the medium already exists in the grid, and sufficient latency for the protection application between the source and HVDC bus can be achieved. Obviously, this must be verified by data transmission tests. The modulated signal is transmitted to, or received from, the power-line by an interfacing circuit, which purpose is to isolate the high voltage of the HVDC bus with the low voltage environment of the PLC. This requirement is challenging in a renewable energy generator based on multi sources or elements, considering that many network nodes are far from the central node, and perhaps serves time-critical applications like those mentioned further below.

Furthermore, while individual nodes communicate only small amounts of data at a time, the total data volume to be transferred through the network is substantial. Hence, resource-eff cient transport of data to and from the central node is mandatory to achieve suff cient network coverage. In PLC networks the communication channel may change abruptly during normal operation. For example, switching operations in medium-voltage energy systems to balance the power consumption by an individual load, storage element or over 
the distribution grid will result in changes of channel transfer functions in sizeable parts of the PLC network. The PLC network design must be able to cope with such abrupt changes, which means that the connectivity must be maintained during or quickly recovered after these changes. In addition, severe network disruptions, as example due to physical removal of network links in case of energy source sensors maintenance or load changes are often not exceptional events but do occur during normal operation in a distributed architecture. Thus a PLC network needs to estimate the qualities of the connections. Indeed, to offer the possibilities of a new adaption of the control system after connections after changes of topology, ad-hoc networking features are needed. It is important to note that the removal and addition of network nodes, or changes in the impedance of the associated device affect the communication channels in a large neighbourhood around this node. Evaluation of any communication technology is only relevant in the context of the operating environment. This seemingly obvious point, frequently bypassed in textbook analysis, cannot be overlooked in the field of power-line communications [11]. The majority of engineering texts heavily relies on the principle of superposition [12]. Unfortunately, the conditions required for superposition to be applicable, i.e. linearity and time invariance are not met for the majority of power-line networks. One cause of nonlinearity is when a packet's signal voltage adds to the DC line voltage and causes power supply electronic switching to turn on and off at the packet carrier frequency. Another cause of possible confusions arises from the common view that wiring capacitance dominates signal propagation effects. This simplified view is rooted in assumptions, which do not accurately reflect power-wiring environments. While it is true that wire capacitance is dominant for cases where the termination or load impedance is much greater than the characteristic impedance of the wire, power-lines are frequently loaded with impedances significantly below the characteristic impedance of the wire. Common examples of loads, which present low network impedance at communication frequencies, include capacitors and inductor used within inverter and battery. The impedance of these devices is typically an order of magnitude, or more, below the characteristic impedance of power wiring.

Thus a standard communication system is built with two main parts, a transmitter and a receiver, both parts can be considered as independent in the conception. Such a system is shown in Fig. 2 for the practical case concerned by the present study. The upper part of this block diagram, Fig. 2(a), is related to the transmitter. In this part, a microcontroller insures the information generation and synchronization. A modulator powered by the microcontroller modulates the signal. The resulting signal is finally amplified before injection, via an interfacing circuit, on the HVDC bus. The lower part of the block diagram, Fig. 2(b), concerns the receiver that has to perform inverse operations than those done by the transmitter. For that, just behind the interfacing circuit a demodulator operates to transfer the demodulated information to the controller. It is to be noted that the microcontroller can also assume the function of maximum power point tracker in the energy production function of the generator. 


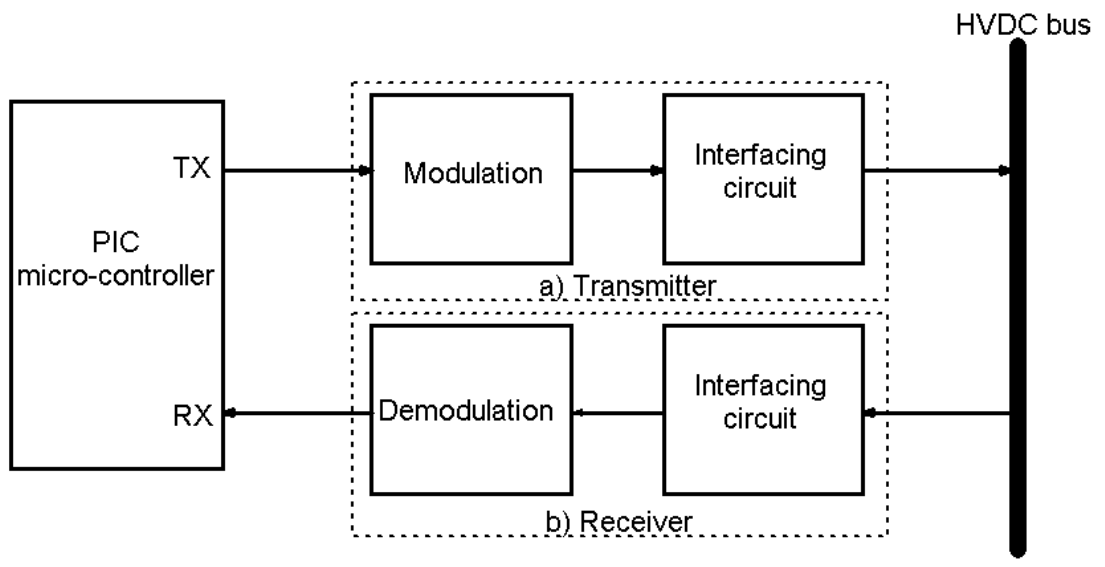

Figure 2: $\quad$ Block diagrams of (a) Transmitter and (b) Receiver of a PLC system.

\section{Design of a master-slave PLC system on HVDC bus for renewable energy generator}

Taking into account the previously presented study and the chosen approach for renewable energy generator, we have developed a PLC system with two different types of circuits for the transmitter-receiver: one, functioning in a slave mode dedicated to the individual optimizers, a second one considered as the master, being the interface with the central management controller. These circuits are presented in Fig. 3 with upper and lower parts for the slave and master circuits, respectively. In the chosen solution, the receiver parts of the slave and master circuits are identical and only the transmitter circuits are different. For experimental validation of the technological choices that we have done in the present study, the HVDC bus voltage is specifically adjusted to $400 \mathrm{~V}$, as determined by the transformation ratio fixed in each individual DC-DC converter. The PIC microcontroller, a PIC16F876 in our application, represented in Fig. 3, is also used for the MPP tracking in the energy conversion part of the step-up DC-DC converter. The PIC microcontroller creates a signal frequency carrier for the controller switch MOSFET M as well as for M11 of the PLC master-slaves circuits. The comparator converts data levels to the reference ones to obtain the two digital levels $0 \mathrm{~V}$ and $5 \mathrm{~V}$.

The main electronic component of the receivers of the PLC master and slave circuits is a transistor mounted in a common emitter mode, which role is to adapt the PLC signal from the HVDC bus to the microcontroller input, pin RA0 of the PIC microcontroller. In this circuit, the received signal enters frst to the demodulator, which recovers the original data. An interfacing branch is used to isolate the receivers from the $400 \mathrm{VDC}$ environment. It is composed by $\mathrm{R} 2, \mathrm{C} 2$ (R22, C22) and combine with the filter circuit L3, C3 (L33, C33). 

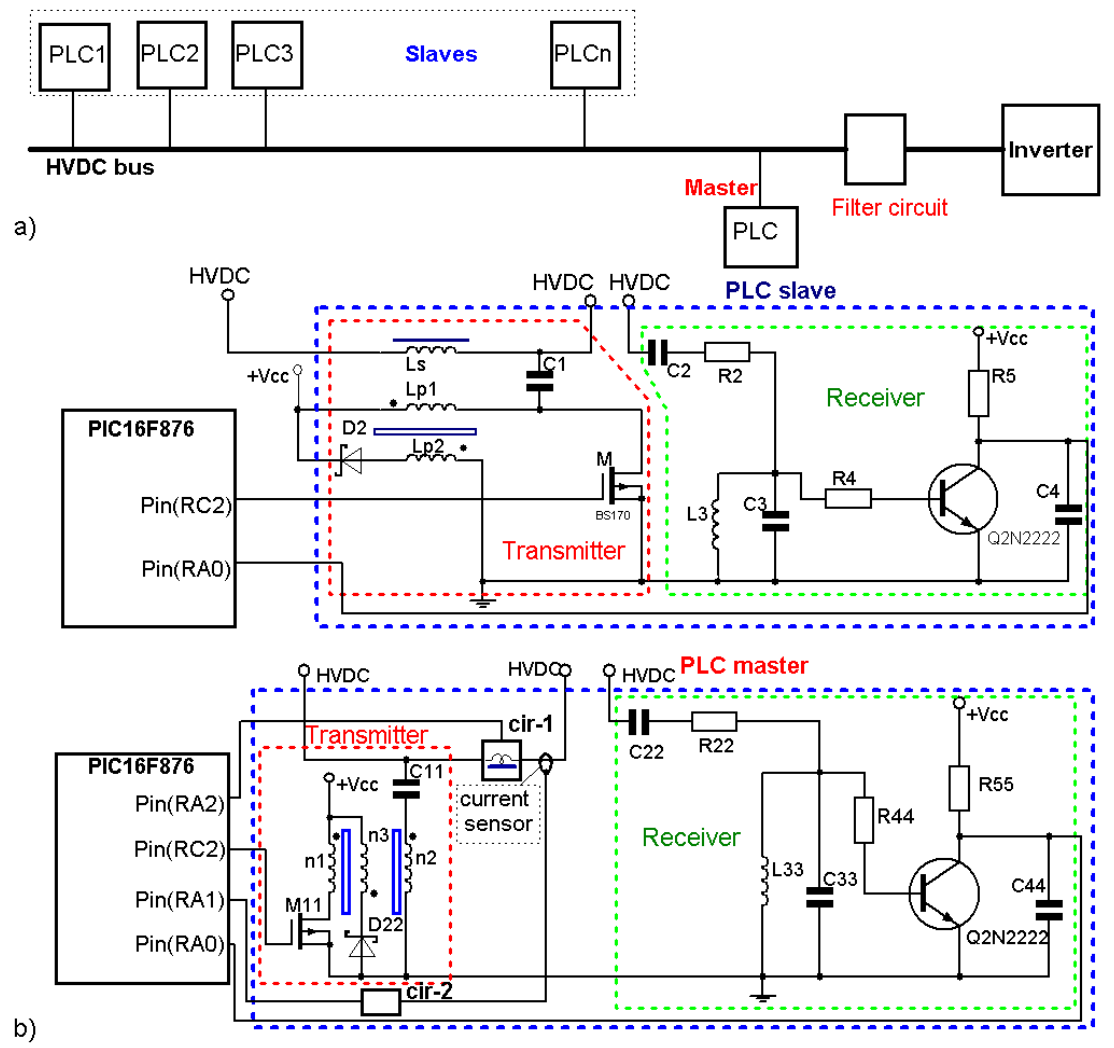

Figure 3: $\quad$ (a) The PLC system master-slave and (b) implementation of the microcontroller for transmitter and receiver parts of PLC master and slave.

In the proposed PLC transmitter part of the slave circuit, the effect of distortion is minimized, by a careful selection of a constant and stable carrier frequency (fc). Therefore, an oscillator is built using the PIC microcontroller generating modulating signal at a frequency of $50 \mathrm{kHz}$. The amplif cation of the signal was designed using a BS170 transistor, dedicated for low-voltage and high-speed applications, especially in inductive circuits. The interfacing circuit consists of a forward-converter transformer where both primary and secondary windings conducting simultaneously with opposing magneto-motive forces along the mutual flux path. The difference of the magneto-motive forces is responsible for maintaining the magnetizing flux in the core. When primary winding current is interrupted by switching off the switch, the dotted ends of the windings develop negative potential to oppose the interruption of current blocking the diode, and thus, interrupting the conduction. To reduce the current delivered by the HVDC bus in the secondary coil, thus avoiding possible saturation, we added, Fig. 3(b), a self, Ls, and a capacitor, C1, in the interfacing circuit of PLC 
system slave. Each PLC slave circuit connected on the HVDC power-line corresponds to a node. The current passing through the PLC transmitter part of the slave circuit is equal the output current of DC-DC converter. The purpose of the Ls coil, Fig. 3(b), is to limit the saturation current in the circuit magnetic of Lp1 - Lp2 transformer. Thus this work design is the same as a forward-converter transformer on the HVDC bus. The current coming from all source-nodes of the HVDC bus crosses the PLC transmitter part of the master circuit. Dimensions of the magnetic circuit in the transformer shown in Fig. 3(b) for the transmitter part are reduced by a combined solution with the resistor of the HVDC line. In addition, to reduce the magnetic-filter circuit, we have chosen a solution integrating two dedicated circuits represented as cir-1 and cir-2 (not detailed in this contribution) in Fig. 3(a). The circuit cir-1 is composed with a switch and a filter. The circuit cir-2 is based on a comparator circuit assuming the comparison between the feedback current provided from the HVDC bus and a fixed but adjustable threshold value. The output of cir- 2 is a voltage proportional to the difference between these two input signals, with its output connected to the microcontroller input RA1.

We present the shape of the various PLC signals occurring in the system: Fig. 4 shows the output simulated information signal as generated by the microcontroller via the transmitter and Fig. 5 shows the PLC signal on the HVDC bus as recorded at the receiver input.

The proposed solution was tested for the validation of the technological choices. The simulation of the optimizers with the PLC circuits was done under Orcad-Proteus environment. The interfacing circuit was tested using three different types of signals: sinusoidal, triangular and rectangular signals to assess the attenuation, distortion and noise performances of the circuit. All signals suffer from high attenuation levels, and among the possible choices, sinusoidal and triangular signals were not used because of the best synchronization possibilities offered by a rectangular waveform in a low signal over noise ratio environment.

The interfacing circuit was tested for information at $50 \mathrm{kHz}$ and for a modulation signal at $500 \mathrm{~Hz}$ as shown in Fig. 4 with amplitude of $5 \mathrm{~V}$. This signal is generated at the pin RC2 of the microcontroller using the Pulse Width Modulation (PWM) method. These choices were done, allowing the use of a band-pass filter at $500 \mathrm{~Hz}$ to limit the influence of high frequency noise as those generated by AM broadcast.

The simulation was performed and the results recorded in three points of the circuit as shown in Figs. 5.

In Fig. 5(a) we can see the signal recorded at a power-line HVDC node. The PLC information signal presents a rectangular shape with amplitude of $+/-5 \mathrm{~V}$ superimposed on the $400 \mathrm{~V}$ of the power HVDC bus. Fig. 5(b) presents the signal at the entry of the receiver part of the slave circuit (see Fig. 3). The frequency on the HVDC power-line is the same as the entering signal, measured at the R2 resistor pins, due to various attenuations, this signal presents a sinusoidal waveform, integrated by the interfacing circuit of the receiver (this 
function is assumed by R2 and C2). Finally, Fig. 5(c) shows the demodulated output signal (Fig. 5(b)) measured at pin RA0 of the controller.

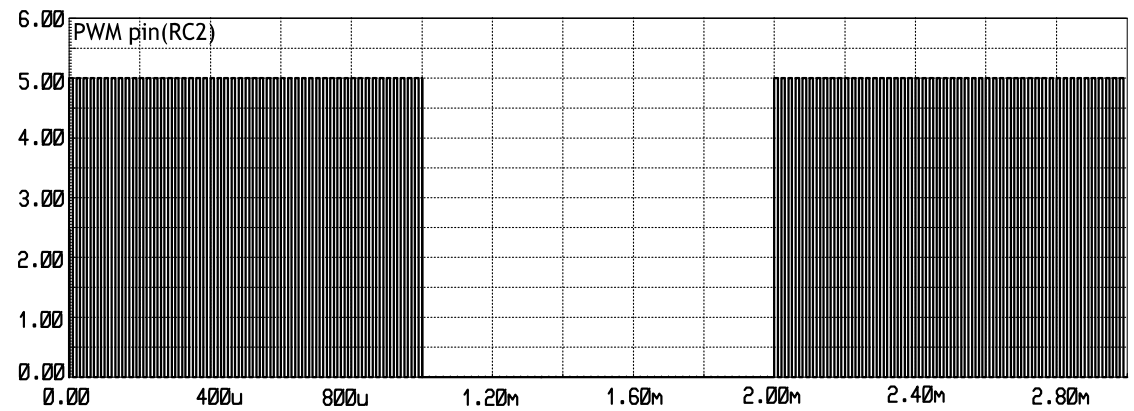

Figure 4: Microcontroller output signal of the MOSFET controller in the PLC slave.
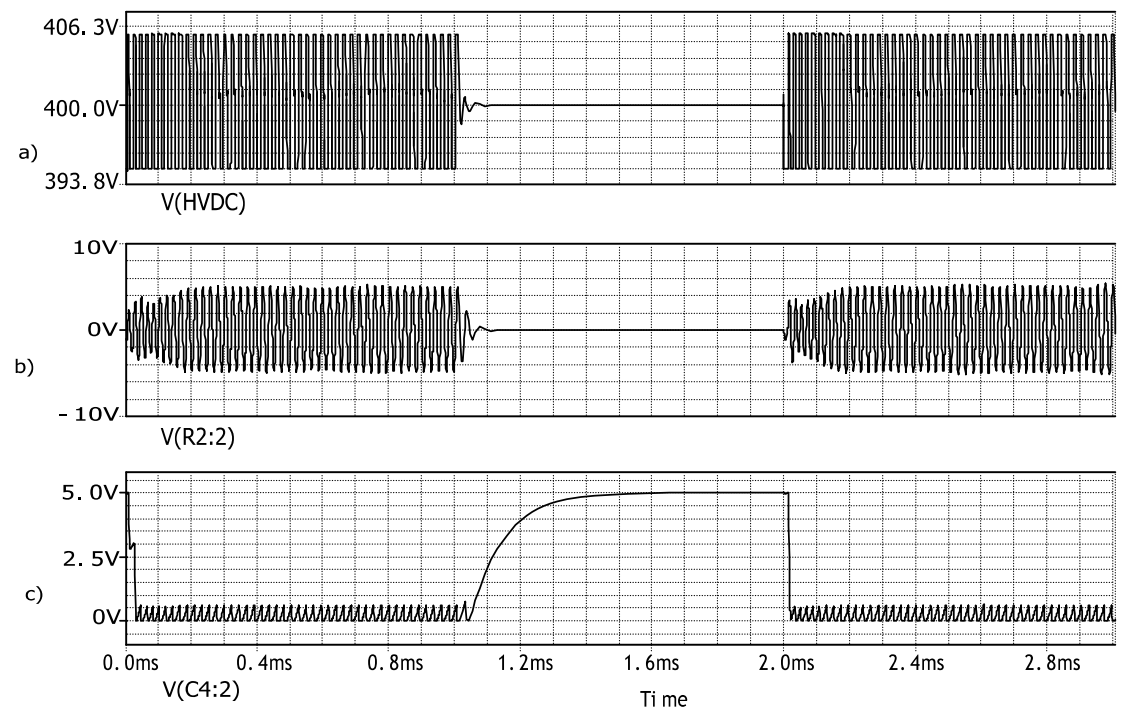

Figure 5: $\quad$ PLC signals (a) on HVDC bus; (b) input of the modulation of receiver; (c) output of demodulation of receiver.

This simulation, based on the specific technology choices done in the present development shows the feasibility of PLC system on HVDC bus for communication of parallel DC-DC optimizers with a central controller in distributed renewable energy production architecture. Especially, the signal shapes presented in Figs 5 show the small influence of the PLC signal on the HVDC bus and the possibility for the PLC circuit to efficiently interface the communication of the optimizer on the bus, with an information signal emitted and transmitted after modulation and finally, correctly detected after demodulation. 


\section{Conclusion}

Initially, this paper has presented how the numerous advantages of the PLC system are developed and associated to a power bus using high DC voltage allowing an improvement of performances and stability of the overall energy transfer process in a renewable energy system. Taking into account these considerations we have designed a simple and reliable PLC master-slave system for DC-DC converters connected to HVDC bus. The system achieves the requirements of stability, reliability, and accuracy. At second, we have simulated the system in continuous operation mode, demonstrating that in the retained solution, the transmitted signal suffered from very low noise levels and distortion. In the framework of smart grid concept, the proposed architecture can be successfully implemented with shelf components to assume low data rate PLC on the HVDC bus allowing applications such as power and remote control, which are necessary in a parallel distributed renewable energy generator system.

\section{Acknowledgement}

The authors gratefully acknowledge Dr. Yves Gillet, Head of the Industrial Engineering and Maintenance Department of the University Institute of Technology, IUT of Thionville-Yutz (France) for financial support and all facilities offered for the present research.

\section{References}

[1] Pinomaa, A., Ahola, J. \& Kosonen, A., Power-line Communication-Based Network Architecture for LVDC Distribution System. IEEE International Symposium on Power-line Communications and Its Applications, pp 358-363, 0,

[2] Nguyen, T.V., Petit, P., Maufay, F., Aillerie, M., \& Charles, J.P., Power-line communication (PLC) on HVDC bus in a renewable energy system. TerraGreen 13, International Conference 2013.

[3] Petit, P., Zegaoui, A., Sawicki, J.P., Aillerie, M., \& Charles, J.P., New architecture for high efficiency DC-DC converter dedicated to photovoltaic conversion. MEDGREEN, Mediteranen, Beyrouth-Liban, 2011.

[4] Vanfretti, L., Hertem, D.V., Nordstrom, L. \& Gjerde, J.O., A Smart Transmission Grid for Europe: Research Challenges in Developing Grid Enabling Technologies. IEEE Transactions on Smart Grid 2011.

[5] Petit, P., \& Aillerie, M., Integration of individual DC/DC converters in a renewable energy distributed architecture. Industrial Technology (ICIT), 2012 IEEE International Conference on, 802-806 (2012) doi: 10.1109/ICIT.2012.6210037, 2012 ICIT Athens Greece. 
[6] Zegaoui, A., Aillerie, M., Petit, P., Sawicki, J.P., Charles, J.P. \& Belarbi, A.W., Dynamic behaviour of PV generator trackers under irradiation and temperature changes. Solar Energy 85, pp. 2953-2964, 2011.

[7] Zegaoui, A., Aillerie, M., Petit, P., Sawicki, J.P., Jaafar, A., Salame, C. \& Charles, J.P., Comparison of Two Common Maximum Power Point Trackers by Simulating of PV Generators. Energy Procedia 6, pp. 678-687 Medgreen 2011, Beyruth, Lebanon, 2011.

[8] Nguyen, T.V., Petit, P., Maufay, F., Aillerie, M., Jafaar, A. \& Charles, J.P., Self-powered high efficiency coupled inductor boost converter for photovoltaic energy conversion. TerraGreen 13, International Conference 2013.

[9] Neha, Shrirao, Ajay, Thakare., Design of Digital Modulators: BASK, BPSK and BFSK using VHDL. International Journal of Advanced Research in Computer Science and Software Engineering, Volume 3, Issue 1, pp. 382-386, 2013.

[10] ABB Review smart grids 2010, report. Available at www.abb.com.

[11] Hrasnica, H., Haidine, A. \& Lehnert, R., Broadband Powerline Communications: Network design. Wiley, 2004.

[12] Roychoudhuri, Ch. \& Tayahi, M., Spectral Super-Resolution by Understanding Superposition Principle and Detecting Processes. IJOMT vol. 1, No. 1, pp. 146-153, 2006. 